\title{
Iterative Tomographic Image Reconstruction Using Fourier-Based Forward and Back-Projectors
}

\author{
Samuel Matej*, Senior Member, IEEE, Jeffrey A. Fessler, Senior Member, IEEE, and Ivan G. Kazantsev, Member, IEEE
}

\begin{abstract}
Iterative image reconstruction algorithms play an increasingly important role in modern tomographic systems, especially in emission tomography. With the fast increase of the sizes of the tomographic data, reduction of the computation demands of the reconstruction algorithms is of great importance. Fourier-based forward and back-projection methods have the potential to considerably reduce the computation time in iterative reconstruction. Additional substantial speed-up of those approaches can be obtained utilizing powerful and cheap off-the-shelf fast Fourier transform (FFT) processing hardware. The Fourier reconstruction approaches are based on the relationship between the Fourier transform of the image and Fourier transformation of the parallel-ray projections. The critical two steps are the estimations of the samples of the projection transform, on the central section through the origin of Fourier space, from the samples of the transform of the image, and vice versa for back-projection. Interpolation errors are a limitation of Fourier-based reconstruction methods. We have applied min-max optimized Kaiser-Bessel interpolation within the nonuniform FFT (NUFFT) framework and devised ways of incorporation of resolution models into the Fourier-based iterative approaches. $\mathrm{Nu}$ merical and computer simulation results show that the min-max NUFFT approach provides substantially lower approximation errors in tomographic forward and back-projection than conventional interpolation methods. Our studies have further confirmed that Fourier-based projectors using the NUFFT approach provide accurate approximations to their space-based counterparts but with about ten times faster computation, and that they are viable candidates for fast iterative image reconstruction.
\end{abstract}

Index Terms-Fast forward and back-projectors, gridding, iterative tomographic reconstruction, min-max interpolation, nonuniform FFT.

\section{INTRODUCTION}

$\mathbf{I}$ TERATIVE image reconstruction algorithms using statistical models of acquired data play an increasingly important role in modern tomographic systems, especially in emission tomography characterized by data with low counts, and consequently, low signal-to-noise ratio [1]-[3]. The computational

Manuscript received May 23, 2003; revised November 12, 2003. This work was supported in part by the National Institutes of Health (NIH) under Grant EB002131 and Grant CA-60711. The Associate Editor responsible for coordinating the review of this paper and recommending its publication was Y. Censor. Asterisk indicates corresponding author.

*S. Matej is with the Department of Radiology, University of Pennsylvania Philadelphia, PA 19104-6021 USA (e-mail: matej@ mipg.upenn.edu).

J. A. Fessler is with the Electrical Engineering and Computer Science Department, University of Michigan, Ann Arbor, MI 48109-2122 USA (e-mail: fessler@umich.edu).

I. G. Kazantsev is with the Department of Radiology, University of Pennsylvania, Philadelphia, PA 19104-6021 USA (e-mail kazantsev@mipg.upenn.edu).

Digital Object Identifier 10.1109/TMI.2004.824233 bottleneck of iterative reconstruction algorithms is the computation of forward and back-projection operations. With the fast increase of the sizes of the tomographic data, reduction of the computation demands of forward and back-projectors is of great importance, as demonstrated by the recent increase of interest in fast computational procedures for calculation of these operations (for example, [4]-[9]). For a family of shift-invariant error norms, another approach [10] uses Fourier methods to combine the forward and backprojection steps into a single operation that can be implemented efficiently with a fast Fourier transform (FFT). Our focus is on positron emission tomography (PET) scans for which Poisson likelihoods or data-weighted least-squares criteria are needed, but these do not belong to the shift-invariant family considered in [10]. Therefore, in the work reported in this paper, we apply Fourier methods to accelerate the forward and backprojection steps separately for iterative tomographic reconstruction. In addition to their computational efficiency, the Fourier-based approaches have potential for additional substantial speed-up by utilizing powerful and cheap off-the-shelf FFT processing hardware.

It has been known for a long time that direct Fourier methods (DFM), that build up the Fourier transform of the object using the Fourier transforms of the projections, have the potential for accurate and high speed reconstruction [11]-[16]. The Fourier-slice theorem was later proposed as a tool for performing the reprojection operation (e.g., [17] and [18]). The crucial step influencing the reconstruction quality and speed is the interpolation between polar and Cartesian rasters in frequency space. Gridding interpolation [19], [20], with proper interpolating [21] and data weighting functions, as investigated in the magnetic resonance imaging literature [22]-[24], brought improvement in the direct Fourier reconstruction. Recently, the Fourier-based reprojection has been applied for (noniterative) fully three-dimensional (3-D) PET reconstruction [25] and for calculation of attenuation correction factors in PET [26]. In these works, Kaiser-Bessel (KB) windows were used for interpolation, which are known to be reasonably accurate, but the accuracy was not evaluated explicitly. The concept of the nonuniform FFT (NUFFT)[27] used in this paper is related to gridding methods for interpolation in frequency space. The KB interpolation kernels used in this work have been optimized using a min-max approach [28], thus providing substantial improvement of the interpolation accuracy.

In the previous works on gridding, the focus was on using the interpolation to find a noniterative approximate solution to an inverse problem. In contrast, we use Fourier-based forward-projection as a tool for calculating the forward problem, and allow iterative reconstruction methods to solve the inverse 
problem. Iterative algorithms need also the ability to compute matrix-vector multiplication by the transpose of the matrix corresponding to forward projection, even though the matrix itself might not be stored explicitly. It is straightforward to reverse (not invert) the steps executed during the forward-projection computation to develop an algorithm to perform multiplication by the transpose, corresponding to the adjoint of the forward operator, which is a form of back-projection. The inverse problem approach used in this paper is also an alternative to gridding in MR image reconstruction [29].

Section II contains descriptions of basic principles of the Fourier-based forward and back-projectors (Section II-A) and of their efficient implementation using NUFFT approach (Section II-B), an outline of the iterative reconstruction approaches using Fourier-based forward and back-projectors (Section II-C), discussion of incorporation of basis functions (Section II-D) and resolution models (Section II-E), and finally discussion of optimized NUFFT interpolation parameters (Section II-F). Results of the numerical error analysis of the NUFFT interpolators based on the min-max methodology are presented in Section III. Effects of interpolation parameters on accuracy of the NUFFT-based forward and back-projectors, as stand alone modules and within iterative reconstruction, are further evaluated using simulated data in Section IV, including performance comparisons of the optimized versions of the Fourier-based forward and back-projectors to their space-based counterparts. Section V contains performance comparisons of the Fourier-based and space-based projectors within iterative reconstruction using physical phantom transmission data acquired on a commercial PET scanner. Finally, discussion and conclusions are in Sections VI and VII.

\section{PRINCIPLES AND IMPLEMENTATION}

\section{A. Fourier-Based Projectors}

Fourier-based forward and back-projectors are based on the central section theorem (also called projection theorem) as outlined in the following. Let $u \in \mathbb{R}^{n}(n=2,3)$ denote a point in the $n$-dimensional image $x(u)$. Let $X(U)$ be the image spectrum, obtained by the $n$-dimensional Fourier transform

$$
X(U)=\int_{\mathbb{R}^{n}} x(u) e^{-i 2 \pi u \cdot U} d u .
$$

Let a straight line in $\mathbb{R}^{n}$ be represented by a direction $\theta \in S^{n-1}$ (unit sphere in $\mathbb{R}^{n}$ ) and a point $u$ as $\left\{u+t \theta: t \in \mathbb{R}^{1}\right\}$. Let $\theta^{\perp} \subset \mathbb{R}^{n}$ represent the subspace orthogonal to the direction $\theta$, that is $\theta^{\perp}=\left\{u \in \mathbb{R}^{n}: u \cdot \theta=0\right\}$, and $r \in \mathbb{R}^{n-1}$ are local coordinates within the subspace $\theta^{\perp}$. Then, the projection (X-ray transform) is defined as

$$
p_{\theta}(r)=\int_{\mathbb{R}^{1}} x\left(u_{\theta, r}+t \theta\right) d t
$$

where $u_{\theta, r}$ are the coordinates in $\mathbb{R}^{n}$ of the point at location $r$ of the subspace $\theta^{\perp}$. The central section theorem [12] is given then by

$$
P_{\theta}(R)=X\left(U_{\theta, R}\right)
$$

where $P_{\theta}(R)$ is the $(n-1)$-dimensional Fourier transform of $p_{\theta}(r)$. Using the central section theorem, the projection at direction $\theta$ and as a function of $r$, can be obtained from the image spectrum $X_{\theta}(R)=X\left(U_{\theta, R}\right)$ by

$$
p_{\theta}(r)=\int_{\theta^{\perp}} X_{\theta}(R) e^{i 2 \pi R \cdot r} d R .
$$

Alternatively, using the same apparatus in reverse, the back-projection can be obtained by depositing the Fourier transform of the projection into the proper locations of the central section of the $n$-dimensional spectral domain, followed by the $n$-dimensional inverse Fourier transform. Note that this backprojection operator is the adjoint of the forward-projection operator employed in the iterative reconstruction approaches, and not the inverse Radon operator used in the direct Fourier or filtered backprojection reconstructions. The inverse Radon operator takes into account additionally the local density of the input data spectrum ("ramp filtering" for the case of the polar spectrum raster).

\section{B. Nonuniform FFT}

Practical implementation of Fourier projectors is based on the discretized version of the projection theorem. The crucial step is obtaining samples of projection spectrum values $\left.P_{\theta}(R)\right|_{R=S \Delta_{R}}$, where $S \in \mathbb{Z}^{n-1}$ ( $\mathbb{Z}$ denotes integer numbers), distributed on the central section planes $\theta^{\perp}$ with grid spacing $\Delta_{R}$ (forming the polar raster in the two-dimensional (2-D) case) from the values of the samples of image spectrum $\left.X(U)\right|_{U=Q \Delta_{U}}, Q \in \mathbb{Z}^{n}$, distributed on the uniform Cartesian raster with spacing $\Delta_{U}$ (forming the rectangular raster in 2-D case). Direct evaluation of image spectrum values at the central section locations using (exact) discrete Fourier transform (DSFT) would require $O\left(N^{4}\right)$ operations for the 2-D image of size $N \times N$. Using NUFFT (related to gridding) allows utilization of fast FT algorithms, thus, substantially speeding-up this process. For the 2-D case, the NUFFT projectors require only $O\left(N^{2} \log N\right)$ operations, compared to $O\left(N^{3}\right)$ needed by the spatial forward-projection algorithms. Basic steps of the NUFFT are as follows:

1) image of size $N$ is first pre-compensated (scaled) for imperfections of the subsequent frequency domain interpolation [19] (step 1 in Fig. 1);

2) calculation of the $K / N$ times oversampled (in each direction) FFT (step 2 in Fig. 1)-image is zeropadded before the FFT (for the efficient implementation of the oversampled FFT, see [13] and [28]);

3) interpolation onto the desired frequency locations within the central section of the spectrum using small local neighborhoods in the frequency domain - this is a crucial operation determining the NUFFT accuracy (step 4 in Fig. 1).

The result of these three steps is the NUFFT, and forward projections are then obtained by performing inverse FFT's on the central section samples (polar lines in 2-D case; step 5 in Fig. 1). The discrete back-projection represents the same set of operations executed in the reverse order. Fourier-based forward and back-projectors for the statistical reconstruction 


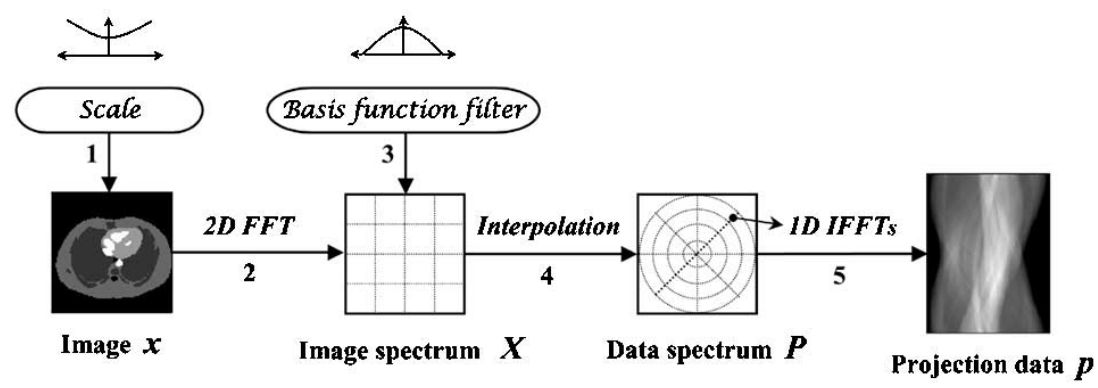

Fig. 1. Basic steps of the NUFFT forward-projection illustrated on the 2-D case: 1) point-wise multiplication of the image with the Scale function-pre-compensation for interpolation imperfections; 2) Fast Fourier Transform on uniform (rectangular) rasters from image into spectrum domain; 3) point-wise spectrum multiplication by Basis function filter-modeling effects of the image representation using basis functions (Section II-D) and of the shift-invariant detector resolution kernels (Section II-E1, for other possibilities of resolution modeling see text); 4) Interpolation into nonuniform (polar) data spectrum raster locations-using the fixed size interpolation kernel; 5) Inverse Fast Fourier Transforms on set of polar lines to obtain a set of projections (sinogram).

techniques should additionally take into account the shape of basis functions used for image representation and resolution properties (e.g., LOR profiles) as described in Sections II-D and II-E (step 3 in Fig. 1).

\section{Fourier-Based Iterative Reconstruction}

Forward and back-projection operations represent the computational bottlenecks within any iterative reconstruction approach. The general flowchart of iterative reconstruction in which the operations of forward and back-projection were substituted by their fast Fourier-based counterparts is depicted in Fig. 2. Specific iterative algorithms will be distinguished one from another by unique discrepancy and update operators. Note that the Fourier-based iterative techniques do not require special treatment of any missing portions of the data, similarly to spatial-based iterative approaches but unlike the transform reconstruction approaches (3-D filtered backprojection method with reprojection (3DRP) [30], 3-D direct Fourier method with Fourier reprojection (3D-FRP) [25]) which do require estimation of missing portions of the data before being employed. In the Fourier-based iterative approaches, the discrepancy operator will provide complete correction data vectors (to be Fourier transformed), including elements having value zero indicating "no-backprojection" in the regions in which data were not measured. These are valid values for the (additive) back-projection operator and result in "no-change" back-projection for the corresponding (image) regions, which is equivalent to "not doing back-projection" for those regions in a typical implementation of the space-based iterative methods.

Within the fast Fourier-based approaches most of the computation time is spent by the calculations of the Fourier transforms on data and image grids. For both forward and back-projection operations of Fourier-based iterative techniques, the (inverse) Fourier transformation of the image (spectrum) has to be done only once per $k$ th image update (i.e., per iteration, or data subset) making it desirable to use large subset sizes (i.e., a low number of subsets) for block-type algorithms. On the other hand, the large subset sizes typically require more passes through the data (iterations). It is easy to show that for linear algorithms the discrepancy operator (based on data difference) and update operator (based on additive operation) can be moved into the Fourier domain, thus eliminating the

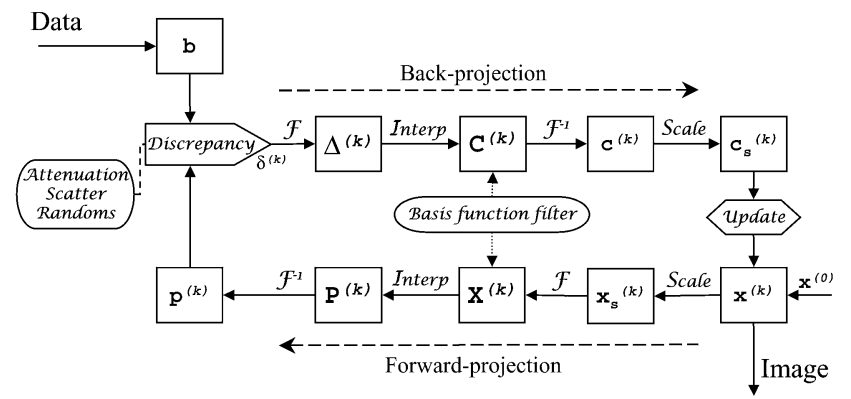

Fig. 2. Flowchart of iterative reconstruction using Fourier-based forward and back-projection. Discrepancy and Update operators are defined by a particular iterative technique. For the 2-D case, the Fourier transformations are 1-D (I)FT of projections on the data side and 2-D (I)FT on the image side. Interpolation operations are performed between data (polar) and image (rectangular) spectrum grids. For Scale and Basis function filter, see caption of Fig. 1.

need to do FFT calculations on image and data rasters at each image update and consequently eliminating the need to use large subset sizes. However, typical statistical reconstruction approaches for emission data are not linear. Fortunately, the speed-up brought by the Fourier-based approaches makes it possible to use increased number of iterations, compared to the space-based approaches, while still providing clinically practical times even for the big subset sizes. Additional substantial speed-up of the Fourier-based approaches can be obtained by using relatively cheap off-the-shelf FFT processor boards.

\section{Emulation of Image Representation Using Basis Functions}

In the conventional space-domain iterative algorithms, the reconstructed image is usually represented by a set of coefficients of basis functions (e.g., pixels, or blobs [31]), rather than by the set of image samples. In this case, the values of the continuous image $x(u), u \in \mathbb{R}^{n}$, can be approximated from coefficients $x_{q}$, where $q$ represents the discrete set of locations $u=q \Delta_{u}$, of basis function $a(u)$ by

$$
x(u) \cong \sum_{q \in \mathbb{Z}^{n}} x_{q} a\left(u-q \Delta_{u}\right) .
$$

If the basis functions are spatially invariant (the typical case), the NUFFT projections through the image composed from those basis functions can be emulated by including a proper spectral filter into the NUFFT path (Basis function filter in Fig. 2). The 
filtering is done simply as multiplication by the basis function spectrum $A(U)$. Among the most popular spatial basis functions are square pixels or rotationally symmetric Kaiser Bessel basis functions (blobs) [31] in the 2-D case and cubic voxels or spherically symmetric blobs in the 3-D case.

\section{E. Resolution Modeling}

A discretized version of the Fourier-section theorem provides discrete samples of the (continuous) projection function $p_{\theta}(r)$ which might be an over-simplified description of the measured data in many tomographic applications. Iterative reconstruction approaches provide convenient ways to include more realistic data acquisition models into their system matrix, such as blurring effects in both sinogram space (e.g., finite detector resolution and crystal penetration) and image space (e.g., positron range). A very useful property of this system matrix is that it can be factored to provide sequence of separate operations modeling individual physical and data acquisition effects [32]. In the following, we describe possibilities of incorporation (in a similar way) of more realistic resolution models into the Fourier-based forward and back-projectors.

1) Shift-Invariant Detector Resolution Model: Assuming that the detector response can be modeled by a shift-invariant blur with impulse response $h(r)$, with corresponding frequency response $H(R)$, the measured data $b$ can be approximated by

$$
\hat{b}_{\theta}\left(s \Delta_{r}\right)=\left(h * p_{\theta}\right)\left(s \Delta_{r}\right)=\int_{\theta^{\perp}} H(R) P_{\theta}(R) e^{i 2 \pi R \cdot s \Delta_{r}} d R
$$

where $\Delta_{r}$ is detector sampling unit, $s \in \mathbb{Z}^{n-1}$. The detector blur can, thus, be modeled by simple multiplication of spectrum of the data, or image, by $H(R)$. Typical examples of $h(r)$ are rect function modeling simple integration over an uniform strip, Gaussian resolution kernel of defined width, or an experimentally obtained resolution kernel.

2) Shift-Variant Detector Resolution Model: The detector resolution function $h_{\theta, s}(r) \equiv h_{\theta}(s, r)$ depends on the detector surface location, i.e., it typically depends on both $\theta$ and $s$. The measured data can be approximated by

$$
\hat{b}_{\theta}\left(s \Delta_{r}\right)=\sum_{s^{\prime} \in \mathbb{Z}^{n-1}} h_{\theta}\left(s^{\prime} \Delta_{r}, s \Delta_{r}\right) \cdot p_{\theta}\left(s^{\prime} \Delta_{r}\right)
$$

using separate resolution kernel function for each projection line location $(\theta, s)$. This operation has to be performed in the projection domain, since it does not have an efficient counterpart in the spectrum domain. Fortunately, the resolution function can usually be approximated by small localized kernels leading to only a minor increase of the computation demands.

3) Image-Space Shift-Variant Resolution Model: This model takes into account those resolution effects which cause variation of the resolution properties throughout the image space, but are independent on angle and location of the projection lines, such as positron range in PET. It is possible that this model might be able to include also other spatially variant resolution properties (similar to deconvolution procedures), but this topic is open for future investigations. Although Fourier-based projectors can not directly take into account the space variant resolution properties, their effect can be modeled in the image domain (rather than during the projection generation process), similarly as it was proposed in [33]. In this case the forward-projection calculation is preceded by blurring of image with the spatially variant resolution kernel $g_{q}(u) \equiv g(q, u)$

$$
\hat{x}\left(q \Delta_{u}\right)=\sum_{q^{\prime} \in \mathbb{Z}^{n}} g\left(q^{\prime} \Delta_{u}, q \Delta_{u}\right) \cdot x\left(q^{\prime} \Delta_{u}\right)
$$

where $\Delta_{u}$ is image sampling unit and $q \in \mathbb{Z}^{n}$. For the back-projection, the blurring operation (with the transpose of the blurring matrix $g$ ) is performed on the correction image $\left(c_{s}^{(k)}\right.$ in Fig. 2) after the back-projection operation and before the update operation. Again, for the small localized resolution kernels this operation represents only a minor increase of the overall computation demands.

\section{F. Min-Max Interpolation Optimization}

The single most important operation within the Fourier-based approaches influencing their quality in a crucial way is the interpolation between the spectrum rasters. In [28], a method was developed for designing and optimization of the finite support interpolators and of the corresponding scaling functions in the min-max sense. The developed min-max analysis provides the interpolator that minimizes the worst case interpolation error over all signals of unit norm. Unfortunately, no analytical formula was found for specifying the optimal choice for the scaling function. Consequently, the space of scaling functions is searched numerically. The true min-max interpolator was further compared to conventional interpolation kernels whose parameters were optimized in the min-max sense. One of the most suitable candidates among them, providing good compromise between accuracy and simplicity, was found to be the KB interpolation kernel. The KB window function has the form [31]

$$
k_{m, J, \alpha}(R)=\frac{1}{I_{m}(\alpha)}\left[\sqrt{1-(2 R / J)^{2}}\right]^{m} I_{m}\left[\alpha \sqrt{1-(2 R / J)^{2}}\right]
$$

for $0 \leq R \leq J / 2$ and value zero for $R>J / 2$, where $R$ is the distance from the KB kernel center, $I_{m}$ denotes the modified Bessel function [34] of order $m, J$ is the size of the KB window and $\alpha$ is a parameter controlling the $\mathrm{KB}$ window shape and frequency characteristics [31], [35] (see examples of KB window functions and of their spectra in Figs. 3 and 4). The interpolation kernel can be given as a radially symmetric KB window function, or as a separable (in spectrum components $R_{1}, R_{2}, \ldots$ ) window function, both of which can be optimized using the same min-max approach. However, although the radially symmetric kernels have very attractive properties if used as basis functions for the image representation within the tomographic reconstruction [31], [35], the separable kernels are preferable, and easier to implement, for the interpolation purposes within NUFFT approaches. The separable KB interpolation kernels used in our studies are given by

$$
k_{m, J, \alpha}\left(R_{1}, R_{2}\right)=k_{m, J, \alpha}\left(R_{1}\right) \cdot k_{m, J, \alpha}\left(R_{2}\right) .
$$




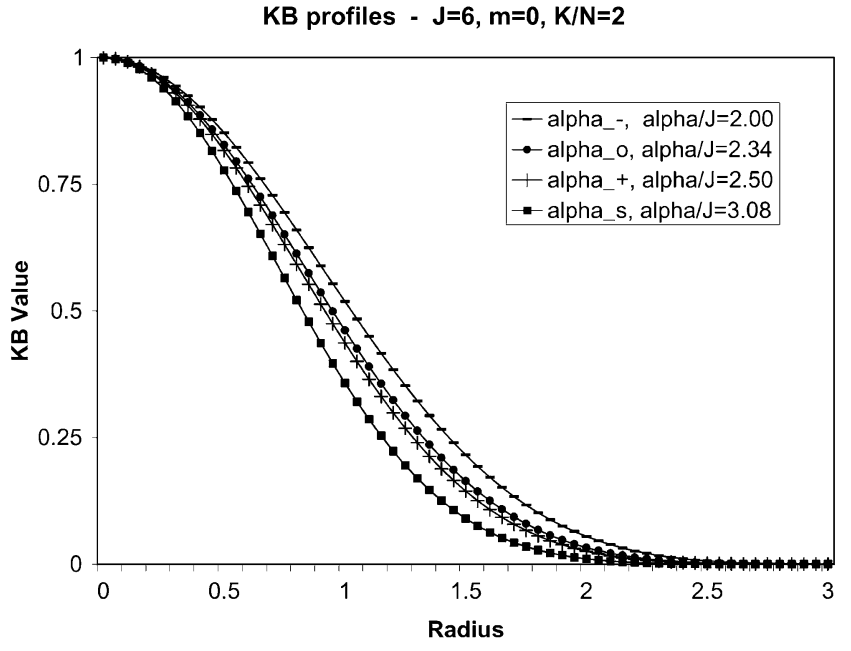

Fig. 3. Profiles of four KB interpolation kernels of size $J=6$ using optimum (alpha_o) and suboptimum parameters for $m=0$ and $K / N=2$. alpha_and alpha_ + represent two suboptimum KB kernels ( $\alpha$ parameter located on both sides from the optimum - star symbols in Fig. 5) providing comparable maximum errors, which are about 6.5 times higher than in the optimum case. For comparison, we show also alpha_s representing typical KB window having desirable properties for the spatial image representation [35], but poor performance as the interpolation kernel. It is interesting that although all of them have similar shape, they provide quite dramatic difference in the NUFFT performance.

We use a scaling function corresponding to the Fourier transform of the KB window. This scaling function gives the lowest worst case error [28], provided the parameters that determine the shape of the KB function were chosen appropriately. In the min-max optimization, the parameters $(\alpha, m)$ of the $\mathrm{KB}$ function are varied by brute-force search, and the values that minimize the worst case error are found numerically for each interpolation kernel size $J$. Based on the results in [28] and on the numerical and experimental results presented in the following we believe that these interpolators are quite close to optimal for the NUFFT problem.

\section{NUMERICAL ERROR ANALYSIS RESUlts}

In [28] it was shown that for the one-dimensional (1-D) NUFFT, a KB window with $m=0$ and $\alpha / J \approx 2.34$ approximately minimizes $E_{\max }$, the worst case interpolation error over all 1-D signals of length $N$ with unit norm. It was further shown that the theoretical error bound of the separable 2-D interpolation kernels is only slightly higher than the worst case error obtained for the 1-D kernels (see formula for the 2-D error in [28]). However, there is no guarantee that a window that is optimized for 1-D worst case error will be optimal for 2-D projection and backprojection with realistic objects. In Section IV, we investigate the effect of the order $m$ and shape parameter $\alpha$ on the accuracy of the 2-D NUFFT step within 2-D forward and backprojection operations for an anthropomorphic object. In this section, we first refine the optimization of $\alpha$ and $m$ for the 1-D NUFFT for comparison to the 2-D case.

We have calculated the maximum error $E_{\max }$ for the range of oversampling factors $(K / N=1,1.5,2,3)$, interpolation kernel sizes $(J=4,5,6,7)$, orders of $\mathrm{KB}$ window $(m \in[-2,2])$ and KB shape (width) parameter $(\alpha$, where $\alpha / J \in[1,3])$. The inter-

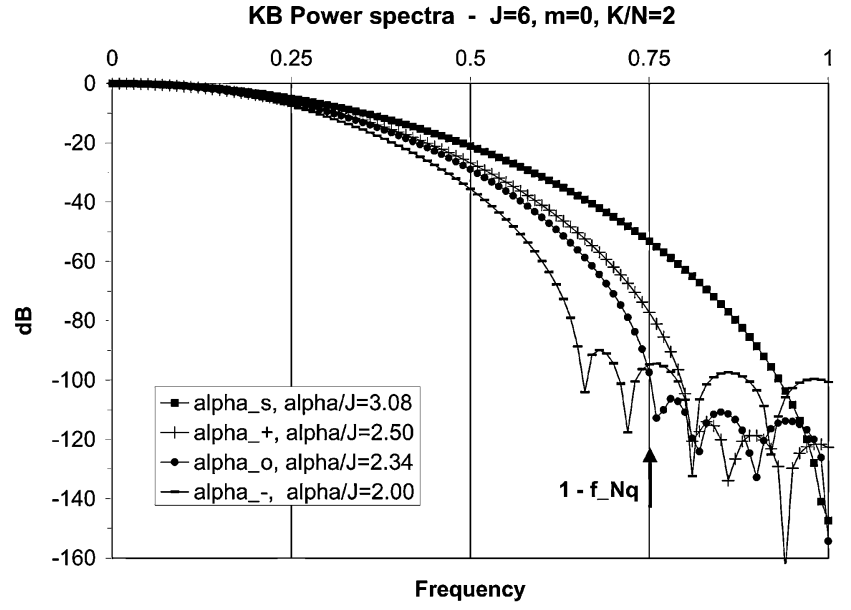

Fig. 4. Power spectra of KB interpolation kernels using optimum (alpha_o) and suboptimum parameters for $J=6, m=0$ and $K / N=2$, whose profiles are shown in Fig. 3.

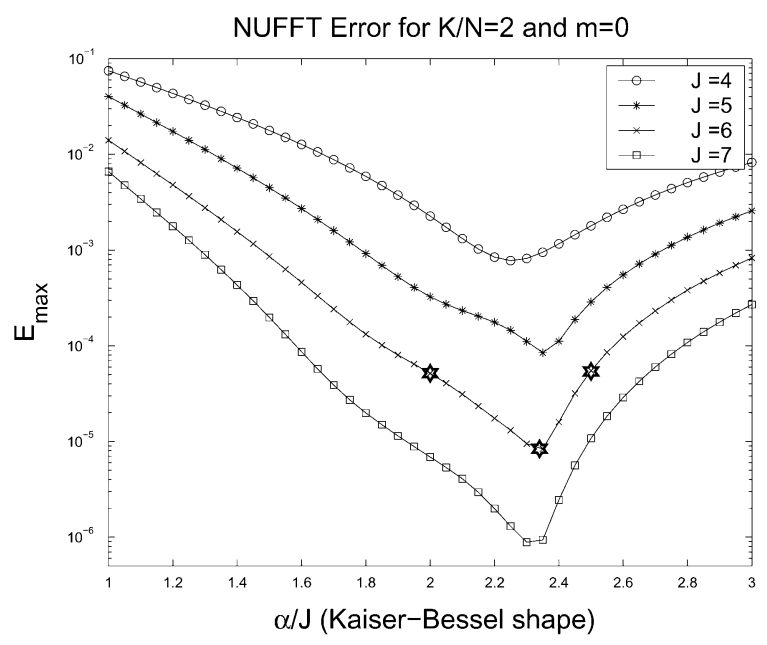

Fig. 5. Maximum error $E_{\max }$ of $\mathrm{KB}$ interpolator as a function of the shape parameter $\alpha$, for several interpolation kernel sizes $J$, Bessel order $m=0$ and using $100 \%$ zero-padding of the spatial domain $(K / N=2)$ (NUFFT interpolator has been found to perform best for the KB orders close to $m=$ 0 -see Fig. 6). Note that the optimum ratio $\alpha / J$ is about 2.34 for various kernel sizes.

polation error is rapidly decreasing with the amount of oversampling. We show results (Figs. 5-7) only for the case $K / N=2$ (a reasonable compromise between the speed and quality) and for the 1-D interpolation window. The behavior for other oversampling cases is similar, as shown in [36]. The optimum order of the $\mathrm{KB}$ interpolator is close to zero for all $K / N$, contrary to our previous experiences with the $\mathrm{KB}$ window used as spatial image basis function [35]. At $m=0$, the optimal values of $\alpha / J$ ratio are approximately constant over a range of KB kernel sizes, but the optimal $\alpha / J$ is different for different oversampling factors (about 1.5 for $K / N=1$, about 2.05 for $K / N=1.5$, about 2.34 for $K / N=2$, and about 2.6 for $K / N=3$ ).

Figs. 3 and 4 show profiles and power spectra, respectively, of optimal and suboptimal interpolation kernels. Note that the interpolation operation is performed in the Fourier domain of the image. Consequently, the image and its periodic repeats (caused by the discretization of the image and Fourier domains) are multiplied by the interpolation kernel spectrum. That means that the 


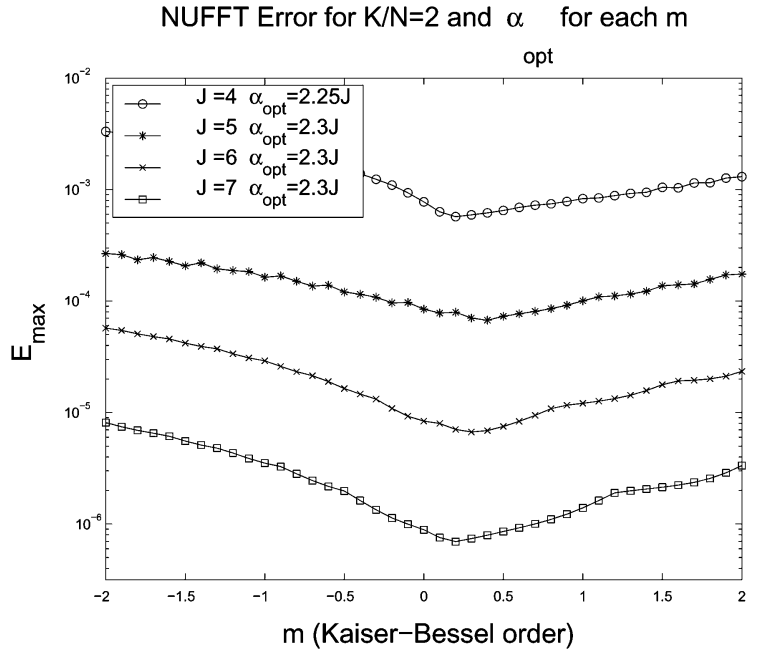

Fig. 6. Maximum error $E_{\max }$ of $\mathrm{KB}$ interpolator as a function of the order $m$, for several sizes $J, 100 \%$ zero-padding $(K / N=2)$ and using optimum ratio $\alpha / J$ for each particular value of $m$. The optimum order parameter $m$ is slightly above 0 for all kernel sizes; $\alpha_{\text {opt }}$ in the legend represent global optimum of the $\alpha$ parameter for the given kernel size.

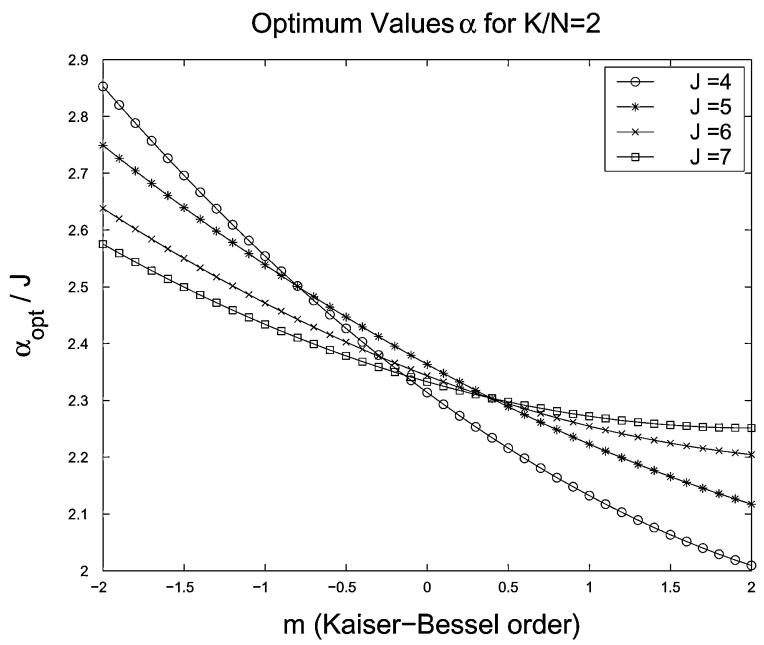

Fig. 7. Values of the optimum ratio $\left(\alpha_{\mathrm{opt}} / J\right)$ as a function of the KB order $m$, for $100 \%$ zero-padding $(K / N=2)$. The values of optimum ratio for individual kernel sizes cluster around similar value for order $m=0$ and diverge for other orders. Similar behavior have been observed for other values of $K / N$, but with different value of the optimum ratio at $m=0$.

kernel spectrum value at the frequency 1.0 (sampling frequency) multiplies the first periodic repeat of the center of the image, and the spectrum value at the frequency $1-f_{-} \mathrm{Nq}$ (periodic repeat of the negative Nyquist frequency $-f_{-} \mathrm{Nq}$ ) multiplies the image value at the periodic repeat of its left boundary. The area between $f \_N q$ and $1-f \_$Nq will not be occupied by either image or its periodic repeat, and its size depends on the size of the oversampling factor $K / N$. The ideal interpolation kernel would suppress all of the image periodic repeats, thus leading to the requirement that it has (effectively) zero value beyond its spectrum frequency $1-f \_$Nq. In practice, the optimum KB interpolation kernel is a compromise between the requirements that the main lobe of its spectrum decays to negligible values at, or before, the image periodic repeat $1-f \_\mathrm{Nq}$ (limiting $\alpha$ from the top) and that its side lobes are effectively zero beyond that point (requiring large $\alpha$ ). Any deviation from this compromise leads to a dramatic increase of the interpolation errors (see star symbols in Fig. 5), in spite of very similar kernel shapes (Fig. 3).

\section{COMPuter Simulation Results}

\section{A. Forward-Projector}

In addition to the numerical evaluation of the NUFFT-based forward projector for the worst case error, we have evaluated the accuracy of the NUFFT-based forward projector using the digital Zubal phantom. We cropped the original $128 \times 128$ image to the size $100 \times 100$ so that the phantom torso fully occupies the whole image region in its wider dimension (see bottom left image in Fig. 11), to avoid any extra zero-padding, other than that given by $K / N$. We have simulated a parallel-beam tomographic system, with a sinogram size of 100 radial bins by 192 angles over $180^{\circ}$, including a rectangular detector response $h(r)=\operatorname{rect}(r)$ with width equal to the pixel size, partially representing the finite detector width in a PET system (rather than using overly idealized line integrals). We have computed forward projections for this system in four ways: using Fourier-based projector with exact (to within double precision in Matlab) evaluation of the 2-D FT (DSFT), using Fourier-based projector with the 2-D NUFFT approximation (to the DSFT) utilizing min-max optimized KB interpolation, using Fourier-based projector with bilinear interpolation, and using space-based projector. Examples of sinograms obtained by Fourier-based and space-based projectors, and corresponding absolute difference images are shown in Fig. 8.

Based on the difference between the exact FT and NUFFT method we have evaluated maximum error, root mean square error, and mean error. In the following graphs, we show only maximum error defined as the maximum absolute difference between exact FT and NUFFT method in percent of the maximum value of the exact FT method. Other errors have been found to exhibit similar behavior, as shown in [36]. The errors have been evaluated for the same set of the NUFFT parameters as in the numerical analysis. The error curves as a function of the $\alpha$ (Fig. 9) show very similar behavior to the numerical case, with nearly exactly the same optima. The optima over $m$ (Fig. 10) are less consistent compared to the theoretical case (Fig. 6) but the locations of the smallest maximum error $E_{\max }$ are still clustered around $m=0$. The calculated sinograms for the optimum values are visually indistinguishable (from the exact FT approach) with errors within $0.06 \%$ when $K / N=2$ even for the smallest kernel size $(J=4)$. By comparison, conventional bilinear interpolation for the Cartesian to polar conversion gives about two orders of magnitude higher maximum error than this small kernel. Table I shows maximum forward-projection errors for optimum shape parameters for different levels of oversampling $K / N$ and different kernel sizes $J$.

\section{B. Back-Projector}

We compared the adjoint operator (back-projector) of the NUFFT-based forward projector using the KB interpolator to the adjoint of the exact Fourier-based reprojector when applied to ramp-filtered ideal sinograms of the Zubal phantom 


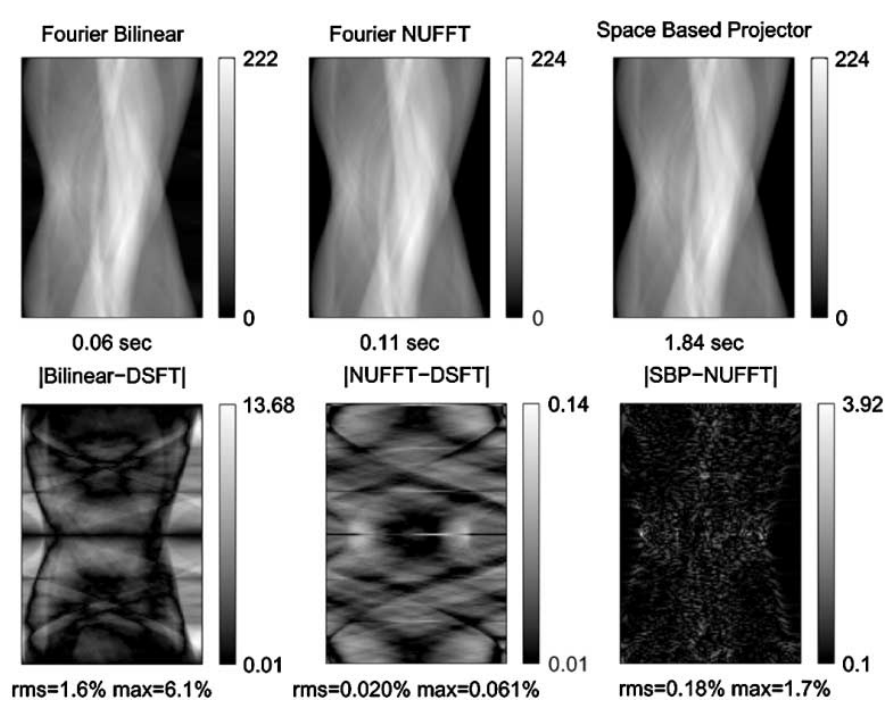

Fig. 8. Example of sinograms (illustrated using 144 angular samples) of Zubal phantom obtained by Fourier-based forward-projector using bilinear interpolation $(K / N=2)$ (top left), Fourier-based forward-projector using NUFFT with optimized KB kernel $(K / N=2, m=0, J=4$, and $\alpha / J=2.4$ ) (top middle) and a space-based forward-projector (SBP) (top right). Illustrative times are for Matlab implementations. Bottom row shows corresponding absolute difference sinograms (including measures of root-mean-square difference and maximum absolute difference) with respect to the exact Fourier projector (DSFT) (bottom left and middle), and Fourier NUFFT projector (bottom right).

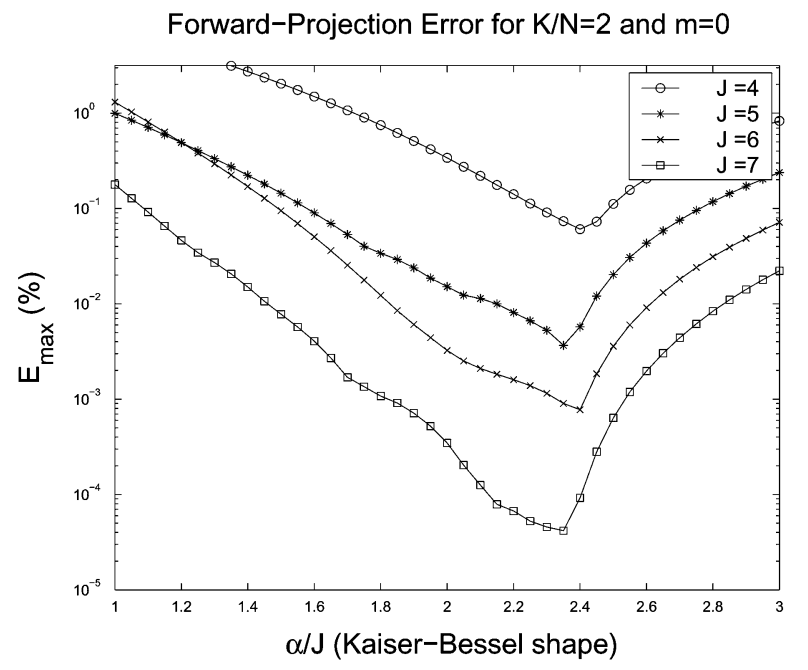

Fig. 9. Maximum interpolation error (\% of projection maximum) of forward-projection of modified Zubal phantom using NUFFT with KB interpolator of several sizes $J$ as a function of the parameter $\alpha$. Same set of parameters used as for the Fig. 5.

of limited size (Fig. 11, bottom left). Examples of images obtained by Fourier-based and space-based back-projectors and corresponding absolute difference images are shown in Fig. 11. Similar to the case of the forward-projector, we have evaluated NUFFT-based back-projector errors for a range of parameters. The maximum errors (shown in graphs in Figs. 12 and 13) have been calculated within the phantom torso region as the percent error of the maximum value in the DSFT images. Again, the error curves are consistent with the previous cases and the NUFFT approach agrees with the exact approach within $0.015 \%$, even for the smallest kernel size $(J=4)$ and

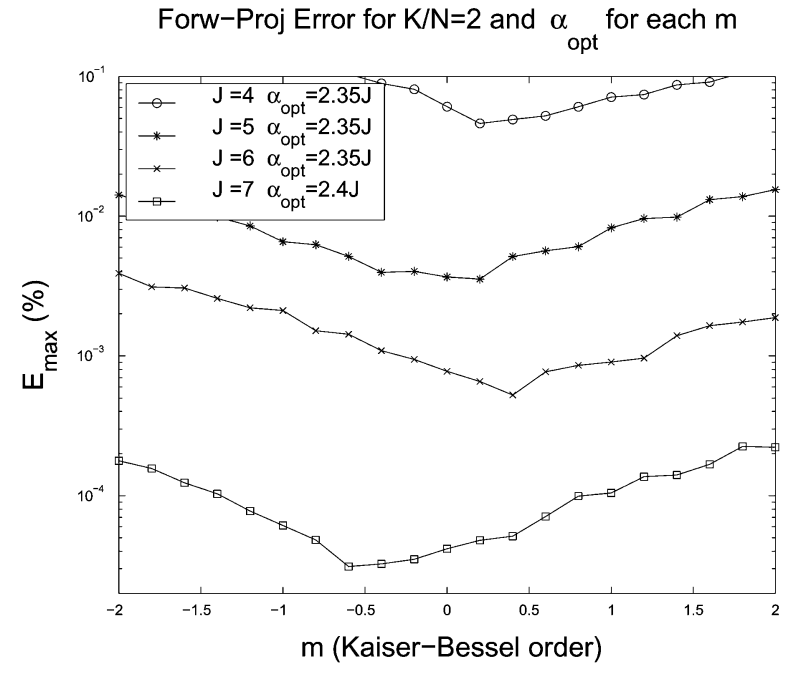

Fig. 10. Maximum interpolation error (\% of projection maximum) of forward-projection of modified Zubal phantom using NUFFT with KB interpolator of several sizes $J$ as a function of the KB order $m$. For each individual $m$ an optimum $\alpha$ was used. $\alpha_{\text {opt }}$ in the legend represent global optimum of the $\alpha$ parameter for the given kernel size.

TABLE I

MAXIMUM FORWARD-PROJECTION ERRORS FOR DIFFERENT OVERSAMPLING AND KERNEL SIZES, USING $m=0$ AND OPTIMUM $\alpha$

\begin{tabular}{lllll}
\hline Oversampling & $\mathrm{J}=4$ & $\mathrm{~J}=5$ & $\mathrm{~J}=6$ & $\mathrm{~J}=7$ \\
\hline $\mathrm{K} / \mathrm{N}=1$ & $5.21 \%$ & $2.27 \%$ & $2.94 \%$ & $1.17 \%$ \\
\hline $\mathrm{K} / \mathrm{N}=1.5$ & $0.11 \%$ & $0.021 \%$ & $0.0039 \%$ & $0.00033 \%$ \\
\hline $\mathrm{K} / \mathrm{N}=2$ & $0.061 \%$ & $0.0037 \%$ & $0.00078 \%$ & $0.000042 \%$ \\
\hline $\mathrm{K} / \mathrm{N}=3$ & $0.033 \%$ & $0.0011 \%$ & $0.00019 \%$ & $0.000007 \%$ \\
\hline
\end{tabular}

$K / N=2$. Table II shows maximum back-projection errors for optimum shape parameters for different levels of oversampling $K / N$ and for different kernel sizes $J$.

\section{Forward and Back-Projector Within Iterative Reconstruction}

Since iterative algorithms require repeated forward and back-projections, it is conceivable that even small errors in the reprojector could accumulate. To study practical performance of the NUFFT forward and back-projector within the iterative reconstruction process, the following experiments have been performed. We have simulated noisy PET sinogram measurements (including attenuation, randoms, and scatter) from the $128 \times 128$ Zubal phantom. We have simulated a parallel-beam tomographic system with a sinogram size of 160 radial bins by 192 angles over $180^{\circ}$. We have run 17 iterations of the conjugate gradient algorithm for a data-weighted least-squares cost function [37] with a standard quadratic first-order roughness penalty. The presented results were obtained using a model of rectangular detector response with a pixel basis function, consistent with the preceding subsections. For the Fourier-based approaches, we have repeated reconstruction studies with a data model involving spatially invariant bell-shaped detector response of equivalent width 

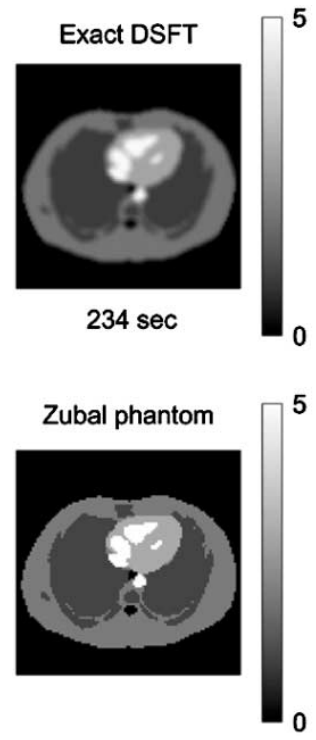
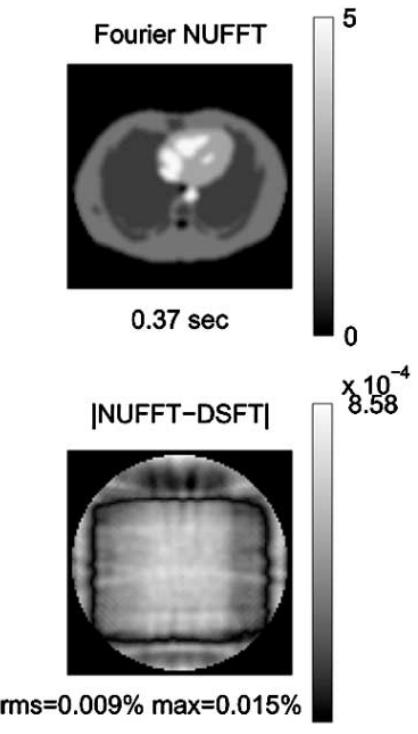
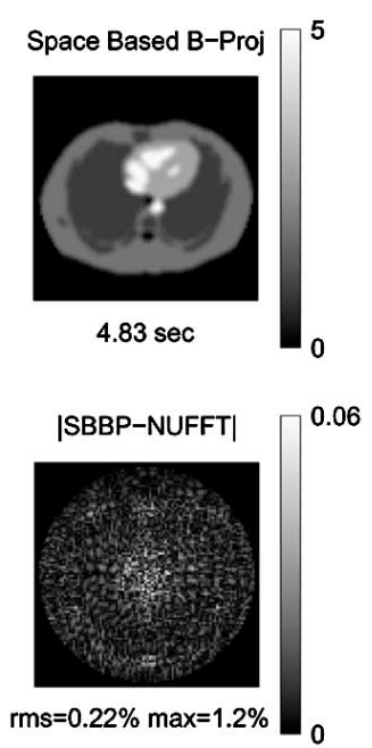

Fig. 11. Example of images obtained by back-projection of filtered sinograms (192 projections) of Zubal phantom (bottom left) using exact Fourier-based back-projector (DSFT) (top left), Fourier-based back-projector using NUFFT with optimized KB kernel $(K / N=2, m=0, J=4$, and $\alpha / J=2.35)$ (top middle) and a space-based back-projector (top right). Illustrative times are for Matlab implementations. Inconsistency between the back-projection times shown here and the corresponding forward-projection times shown in Fig. 8 is caused by the memory access ordering in the Matlab implementation. Back-projection and forward-projection would take about the same time with special-purpose implementations. Bottom (middle and right) row shows corresponding absolute difference images (including measures of root-mean-square difference and maximum absolute difference) with respect to the exact Fourier back-projector (DSFT) (bottom middle), and Fourier NUFFT projector (bottom right).

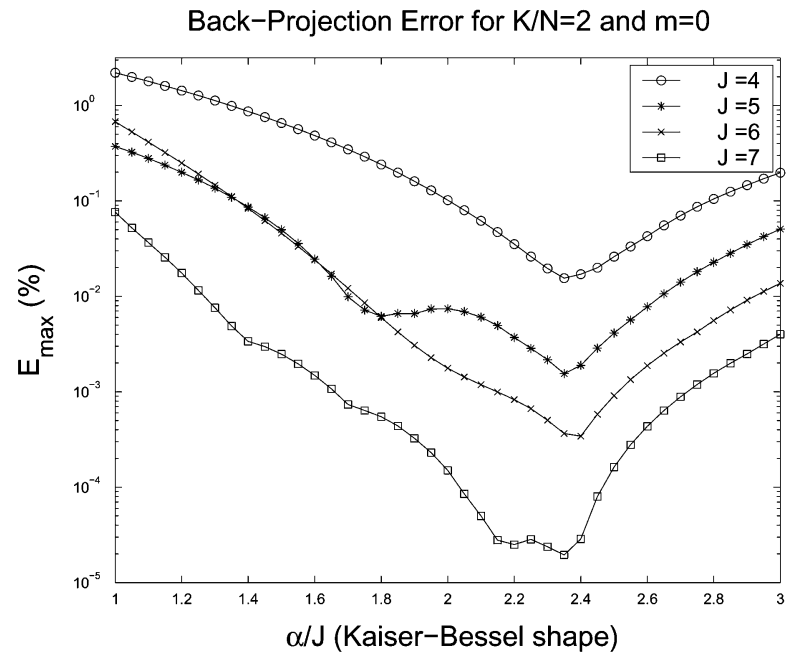

Fig. 12. Maximum interpolation error (\% of phantom maximum) of discrete back-projection using NUFFT with KB interpolator of several sizes $J$ as a function of the parameter $\alpha$. Same set of parameters used as for the Figs. 5 and 9.

to the image grid size and modeling image representation by smooth (blob) basis functions. Examples of reconstructed images using Fourier-based and space-based forward and back-projectors and corresponding absolute difference images are shown in Fig. 14. The reconstructed images using DSFT, NUFFT and space-based projectors with pixel basis functions (top row) are visually indistinguishable. Reconstructions with an image model involving smooth basis functions (illustrated at the bottom left) provide decreased noise levels, as expected.

The errors of NUFFT-based forward and back-projectors within the iterative reconstruction, as compared to the reconstruction using exact FT projectors (DSFT), have been evaluated for the same set of parameters as in the previous

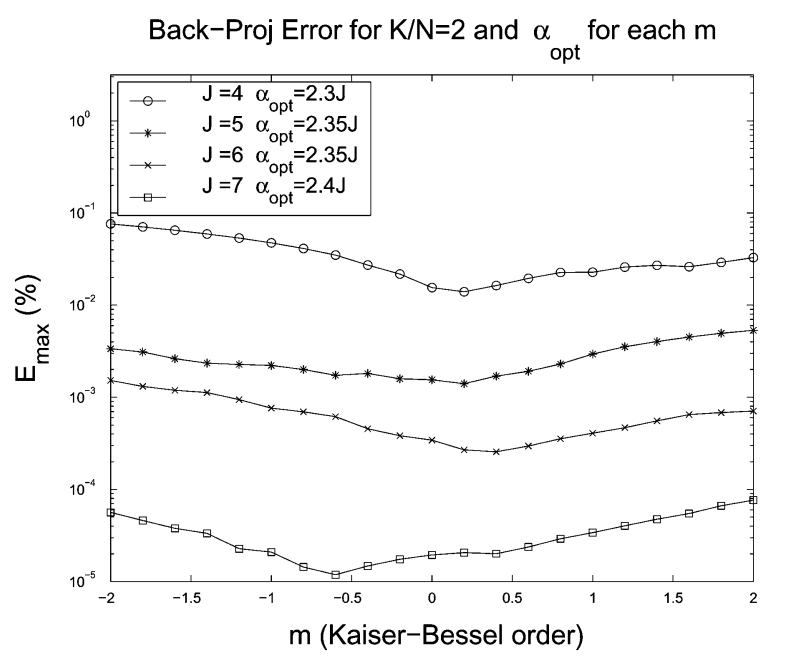

Fig. 13. Maximum interpolation error (\% of phantom maximum) of discrete back-projection using NUFFT with KB interpolator of several sizes $J$ as a function of the KB order $m$. For each individual $m$ an optimum $\alpha$ was used. $\alpha_{\text {opt }}$ in the legend represent global optimum of the $\alpha$ parameter for the given kernel size.

TABLE II

MAXIMUM BACK-PROJECTION ERRORS FOR DIFFERENT OVERSAMPLING AND KERNEL SIZES, USING $m=0$ AND OPTIMUM $\alpha$

\begin{tabular}{lllll}
\hline Oversampling & $\mathrm{J}=4$ & $\mathrm{~J}=5$ & $\mathrm{~J}=6$ & $\mathrm{~J}=7$ \\
\hline $\mathrm{K} / \mathrm{N}=1$ & $9.10 \%$ & $1.32 \%$ & $1.75 \%$ & $0.71 \%$ \\
\hline $\mathrm{K} / \mathrm{N}=1.5$ & $0.099 \%$ & $0.020 \%$ & $0.0042 \%$ & $0.00068 \%$ \\
\hline $\mathrm{K} / \mathrm{N}=2$ & $0.015 \%$ & $0.0015 \%$ & $0.00034 \%$ & $0.000019 \%$ \\
\hline $\mathrm{K} / \mathrm{N}=3$ & $0.0075 \%$ & $0.00044 \%$ & $0.000063 \%$ & $0.000002 \%$ \\
\hline
\end{tabular}

cases. The maximum error has been calculated within the phantom torso region and expressed as the percent error 

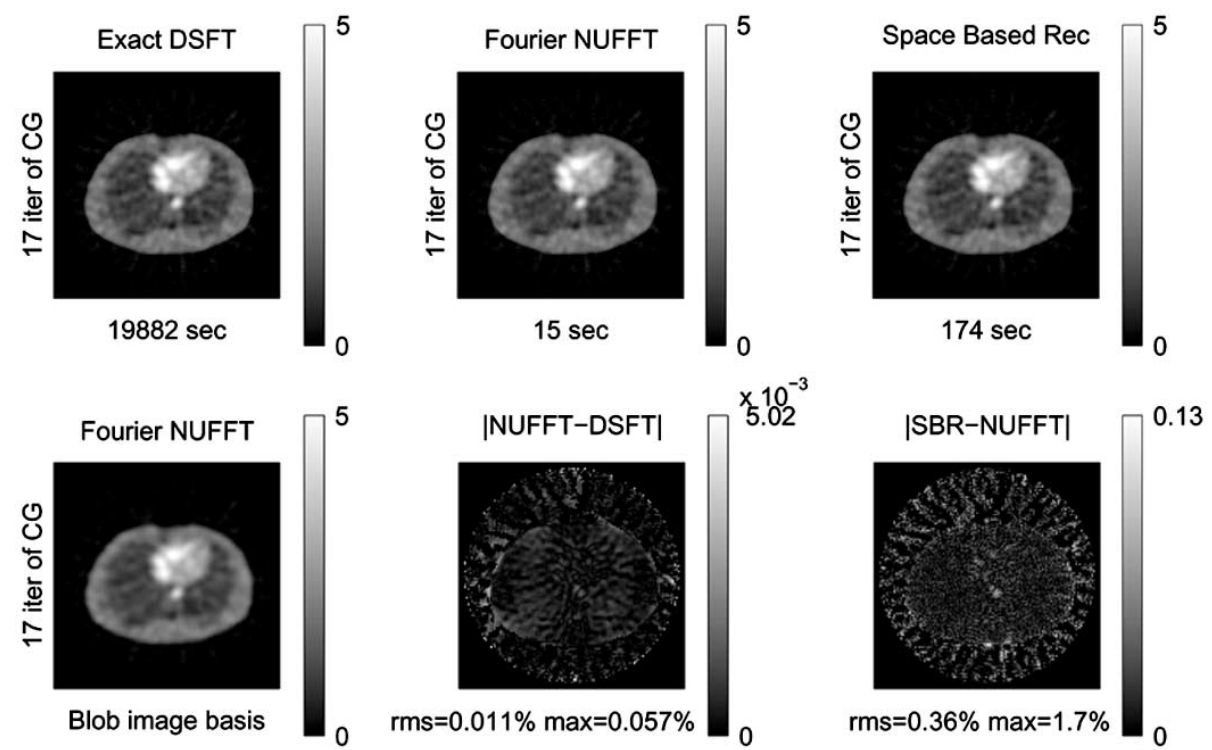

Fig. 14. Example of QPWLS-CG reconstructions (17 iterations) of thorax phantom using exact DSFT (top left), Fourier-based NUFFT with optimized KB kernel $(K / N=2, m=0, J=4$, and $\alpha / J=2.55)$ (top middle) and space-based (SBR) (top right) forward and back-projectors. Illustrative times are for Matlab implementations. Bottom left is illustration of NUFFT iterative reconstruction including modeling of a blob basis function and bell-shaped detector resolution kernel. Bottom (middle and right) row shows corresponding absolute difference images (including measures of root-mean-square difference and maximum absolute difference) with respect to the exact Fourier projectors (DSFT) (bottom middle), and Fourier NUFFT projectors (bottom right).

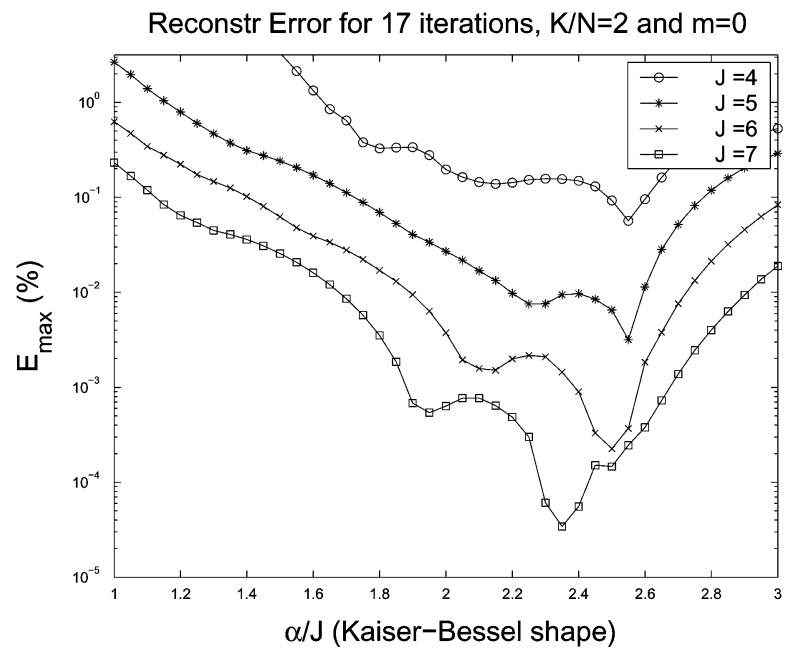

Fig. 15. Maximum interpolation error (\% of phantom maximum) of 17 iterations of QPWLS reconstruction using NUFFT forward and back-projectors with KB interpolator of several sizes $J$ as a function of the parameter $\alpha$. Same set of interpolation parameters used as for the Figs. 5, 9, and 12.

relative to the maximum value in the phantom. The error curves (Figs. 15 and 16) show again similar behavior, with the optimum slightly shifted toward higher parameter $\alpha$ values. This is probably caused by the fact that the phantom does not cover the whole image region (essentially constituting additional zero-padding). The maximum error is below $0.06 \%$ for the smallest kernel size $(J=4)$ and $K / N=2$. Table III shows the maximum reconstruction errors for optimum shape parameters for different levels of oversampling $K / N$ and for different kernel sizes $J$. Fourier-based reconstructions with an image model involving smooth basis functions showed similar comparisons with slightly decreased errors, as shown in [36].

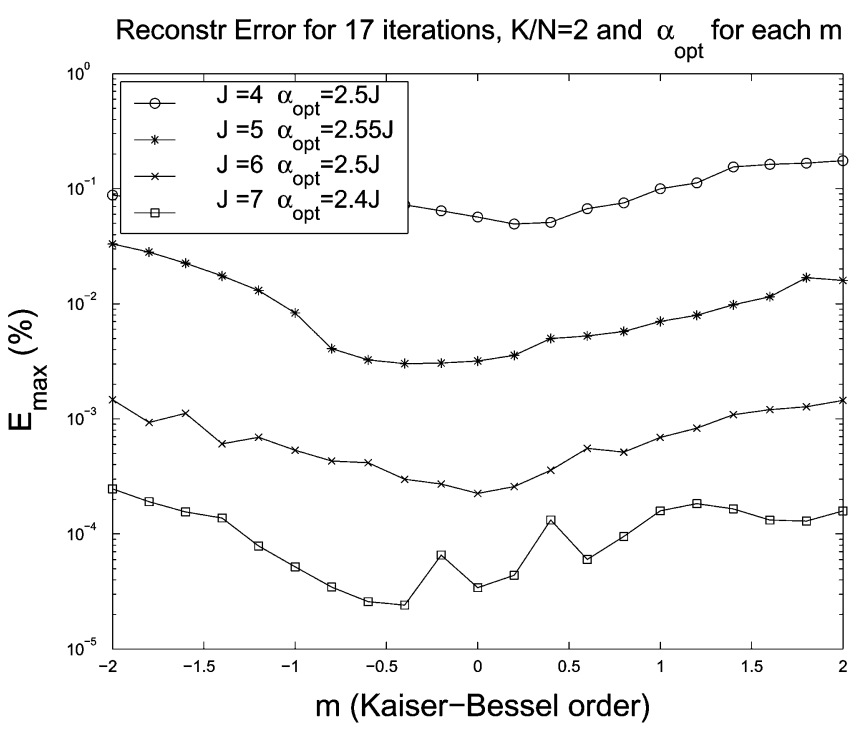

Fig. 16. Maximum interpolation error (\% of phantom maximum) of 17 iterations of QPWLS reconstruction using NUFFT forward and back-projectors with KB interpolator of several sizes $J$ as a function of the KB order $m$. For each individual $m$ an optimum $\alpha$ was used. $\alpha_{\text {opt }}$ in the legend represent global optimum of the $\alpha$ parameter for the given kernel size.

TABLE III

MAXIMUM RECONSTRUCTION ERRORS FOR DIFFERENT OVERSAMPLING AND KERNEL SIZES, USING $m=0$ AND OPTIMUM $\alpha$

\begin{tabular}{lllll}
\hline Oversampling & $\mathrm{J}=4$ & $\mathrm{~J}=5$ & $\mathrm{~J}=6$ & $\mathrm{~J}=7$ \\
\hline $\mathrm{K} / \mathrm{N}=1$ & $0.59 \%$ & $0.23 \%$ & $0.056 \%$ & $0.031 \%$ \\
\hline $\mathrm{K} / \mathrm{N}=1.5$ & $0.098 \%$ & $0.0081 \%$ & $0.0011 \%$ & $0.00055 \%$ \\
\hline $\mathrm{K} / \mathrm{N}=2$ & $0.057 \%$ & $0.0032 \%$ & $0.00023 \%$ & $0.000034 \%$ \\
\hline $\mathrm{K} / \mathrm{N}=3$ & $0.039 \%$ & $0.0020 \%$ & $0.00010 \%$ & $0.000010 \%$ \\
\hline
\end{tabular}



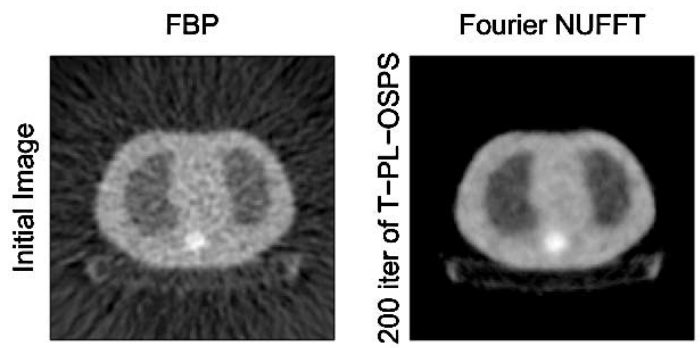

$206 \mathrm{sec}$

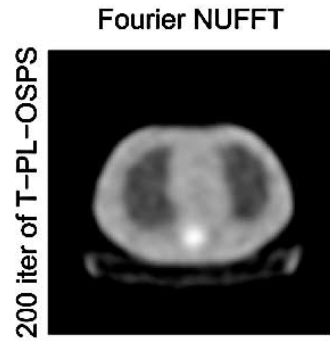

Blob image basis
|NUFFT-SBR|

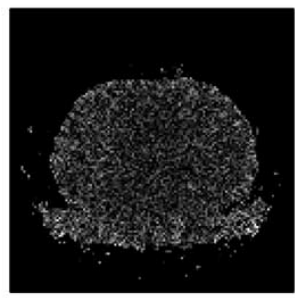

$\mathrm{rms}=0.15 \% \max =1.29 \%$

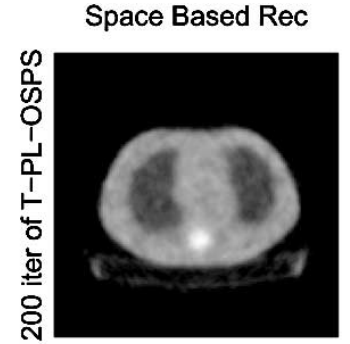

$1659 \mathrm{sec}$

Fig. 17. FBP (serving as initial image) (top left) and T-PL-OSPS reconstructions (200 iterations) from transmission data (from ECAT-921 scanner) of physical thorax phantom using Fourier-based NUFFT with (theoretically) optimized KB kernel $(K / N=2, m=0, J=4$, and $\alpha / J=2.34$ ) (top middle) and space-based (SBR) (top right) forward and back-projectors. Illustrative times are for Matlab implementations. Bottom left is illustration of NUFFT iterative reconstruction including modeling of a blob basis function and bell-shaped detector resolution kernel. Bottom right is absolute difference image (including measures of root-mean-square difference and maximum absolute difference) between reconstructions using Fourier-based NUFFT and space-based projectors.

\section{ITERATIVE RECONSTRUCTION USING REAL DATA}

The performance of the Fourier-based forward and backprojectors within iterative reconstruction has been further tested (and compared to the space-based projectors) using real PET data. For this study, we have used transmission data of a physical torso phantom acquired on the clinical scanner ECAT-921. The data contained 160 radial bins by 192 angles over $180^{\circ}$, with projection ray size $3.38 \mathrm{~mm}$ and reconstructed image pixel size $4.22 \mathrm{~mm}$. The attenuation image has been reconstructed using 200 iterations of the transmission penalized-likelihood algorithm T-PL-OSPS [38] (with number of subsets equal to one) initialized by the filtered-backprojection image (shown at top left in Fig. 17). Although the number of iterations used in practice would be much lower, we have run the algorithms for 200 iterations to test if there is any accumulation of errors or any instability in the Fourier-based approach, as the iterations progress. The Fourier-based approach showed stable behavior consistent with the space-based approach. The observed measures of the difference between the two approaches did not change by more than $1 \%$ (of their respective maximum values at iteration 200, reported in Fig. 17) during the last 110-120 iterations.

Examples of reconstructed images using Fourier-based and space-based forward and back-projectors and a corresponding absolute difference image are shown in Fig. 17. Horizontal profiles through the center part of the reconstructed images are shown in Fig. 18. The reconstructed images using NUFFT and space-based projectors with pixel basis functions (Fig. 17 top middle and right, Fig. 18 solid line profiles) are visually indistinguishable. Reconstructions with an image model involving smooth basis functions (illustrated in Fig. 17 at the bottom left) provide decreased noise levels while preserving the edges (see dashed line profile in Fig. 18).

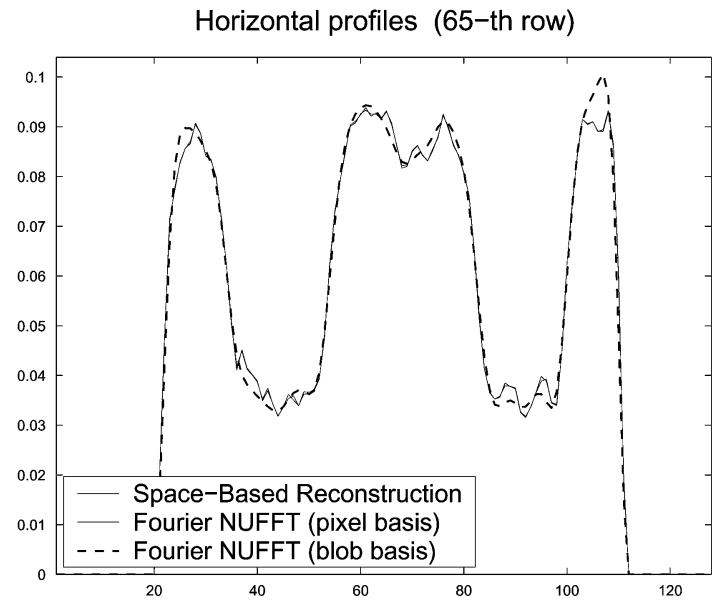

Fig. 18. Horizontal profiles through the iterative reconstructions shown in Fig. 17. Space-based and Fourier-based reconstructions using pixel basis functions (solid lines) are closely overlapping. Fourier-based reconstruction modeling blob basis function (dashed line) provides lower noise levels (while preserving edges), in agreement with our previous experiences with (space-based) iterative reconstructions using blob basis functions [35].

\section{DISCUSSION}

The results reported within this paper were obtained for the 2-D case. The illustrative computation times reported in the figures are for Matlab implementations. The Fourier-based forward and back-projectors were found to be more than 10 times faster compared to their space-based counterparts (see Figs. 8 and 11). Similar speed-up is expected for optimized special-purpose implementations of both approaches. The Fourier-based approaches can be straightforwardly extended to the 3-D case as was done for the 3-D version of direct Fourier method (3D-FRP [25]), which involved both back-projection and forward-projection (reprojection) operations. Extrapolating 
from experience with the 3D-FRP [25], the fully 3-D iterative approaches using Fourier-based projectors will have the potential to speed-up the reconstruction time about 5-10 times for images of size $128^{3}$, and this speed-up will be increasing with the image size. An additional substantial speed-up of Fourier-based approaches is feasible using relatively cheap off-the-shelf FFT processor boards. The speed-up of the reconstruction approaches is very important, as supported by the observations [6] that the data volumes in modern PET systems might be increasing at a faster rate than the increase of computer power as described by Moore's law.

It is worth mentioning that the two Fourier-based reconstruction approaches mentioned above (3D-FRP and iterative), both use back-projection and forward/reprojection operations and, thus, both benefit considerably from the Fourier-based forward and back-projectors, but the two approaches are quite distinctive in nature. 3D-FRP is based on the discretized inverse Radon formula derived for the ideal continuous model and the image is obtained in one pass through the data which are weighted in the frequency domain for the sampling density of the data spectrum and for nonuniformities introduced by the interpolation. On the other hand, the Fourier-based iterative approaches, which are the focus of this paper, are derived based on a discrete image and data acquisition model while taking into account data statistics. Here, the image is gradually built-up and/or refined (based on particular discrepancy and update operations) through an iterative process, and the image update step is based on the simple back-projection (without data filtering) which is an adjoint operation to the forward projection.

Direct application of the NUFFT approach is limited to uniformly spaced parallel projection data. However, it can be easily extended to fan beam, cone beam, or any other kind of data that can be resorted into sets of parallel lines with nonequidistant spacing. In this case, by using the duality principle, the nonuniform raster is defined by the distribution of the parallel projection lines for each direction $\theta$ and the NUFFT output is the uniform spectral raster of the projection data on $\theta^{\perp}$. This operation (or its adjoint) replaces the operation of (I)FFT of projections within the NUFFT back (forward)-projectors described in Section II-B. On the other hand, it should be mentioned that the Fourier-based approaches are not applicable to the unsorted (e.g., list-mode) data.

A noteworthy property of the Fourier-based approaches is that they can be straightforwardly applied to the case of data and/or image defined on the efficient spatial grids (hexagon in 2-D case and body-centered cubic grid in 3-D case [39]) thanks to the existence of efficient FFT algorithms for those grids.

Finally, it is important to emphasize that we have been utilizing KB window functions in two quite distinct ways within the framework of the Fourier-based iterative approaches. First, the KB window has been utilized as the localized interpolation kernel in the spectrum-domain interpolation-the crucial NUFFT operation. Second, it has been used in the optional operation of modeling of the spatial-domain image basis function. These are independent operations having quite different requirements on the KB window shape, as illustrated in Section III.

\section{CONCLUSION}

Our results show very good agreement of the theoretical min-max error analysis of the NUFFT forward and back-projectors with their practical performance. Consequently, the min-max approach offers a valid and practical framework for the optimization of the NUFFT interpolation parameters.

Our results further show that the NUFFT-based forward and back-projectors with the min-max optimized KB interpolation are fast and very accurate. In particular, their approximation errors have been found to be extremely low as compared to the exact discrete Fourier transform approach, and they have manifested a very good match to the space-based projectors, even for small oversampling and interpolation kernel sizes. For example, it has been observed that for the optimized KB interpolators it might be sufficient to use just 50\% FFT oversampling and the interpolation kernels of diameter spanning just 4 to 5 grid points.

In summary, it has been demonstrated that the Fourier-based forward and back-projectors utilizing the NUFFT approach provide fast and extremely accurate tools for iterative tomographic reconstruction. The Fourier-based projectors are especially attractive for the fully 3-D iterative reconstruction approaches in PET characterized by very large data volumes. An additional advantage of the Fourier-based approaches is the possibility of utilizing the powerful and cheap off-the-shelf FFT processing hardware.

\section{ACKNOWLEDGMENT}

The authors would like to thank the reviewers for their detailed comments, and gratefully acknowledge R. M. Lewitt for fruitful discussions and comments on this work.

\section{REFERENCES}

[1] R. M. Leahy and J. Qi, "Statistical approaches in quantitative positron emission tomography," Statistics and Computing, vol. 10, no. 2, pp. $147-165,2000$.

[2] J. A. Fessler. (2001) Statistical Image Reconstruction. [Online]. Available: http://www.eecs.umich.edu/ fessler/

[3] R. M. Lewitt and S. Matej, "Overview of methods for image reconstruction from projections in emission computed tomography," Proc. IEEE, vol. 91, pp. 1588-1611, Oct. 2003.

[4] S. Basu and Y. Bresler, " $O\left(N^{2} \log _{2} N\right)$ filtered backprojection reconstruction algorithm for tomography," IEEE Trans. Image Processing, vol. 9 , pp. $1760-1773$, Oct. 2000.

[5] J. Hamill, C. Michel, and P. Kinahan, "Fast PET EM reconstruction from linograms," IEEE Trans. Nucl. Sci., vol. 50, pp. 1630-1635, Oct. 2003.

[6] D. Brasse, P. E. Kinahan, R. Clackdoyle, C. Comtat, M. Defrise, and D. W. Townsend, "Fast fully 3-D image reconstruction using planograms," IEEE Trans. Med. Imag., pp. 413-425, Apr. 2004.

[7] B. De Man and S. Basu. Distance-driven projection and backprojection for computed tomography. presented at Proc. 2002 IEEE Nuclear Science Symp/Medical Imaging Conf. [CD-ROM] M10-89

[8] H. Zhao and A. J. Reader. Fast projection algorithm for voxel arrays with object dependent boundaries. presented at Proc. 2002 IEEE Nuclear Science Symp/Medical Imaging Conf. [CD-ROM] M10-104

[9] A. Averbuch, R. R. Coifman, D. L. Donoho, M. Israeli, and J. Waldén, "Fast slant stack: A notion of Radon transform for data in a Cartesian grid which is rapidly computable, algebraically exact, geometrically faithful and invertible," SIAM Scientific Computing, 2004, to be published.

[10] A. H. Delaney and Y. Bresler, "A fast and accurate Fourier algorithm for iterative parallel-beam tomography," IEEE Trans. Image Processing, vol. 5, pp. 740-753, May 1996.

[11] R. M. Lewitt, "Reconstruction algorithms: Transform methods," Proc. IEEE, vol. 71, pp. 390-408, Mar. 1983. 
[12] A. C. Kak and M. Slaney, Principles of Computerized Tomographic Imaging. Piscataway, NJ: IEEE Press, 1987.

[13] N. Niki, R. T. Mizutani, Y. Takahashi, and T. Inouye, "A high-speed computerized tomography image reconstruction using direct two-dimensional Fourier transform method," Syst. Comput. Controls, vol. 14, no. 3, pp. 56-65, 1983 .

[14] S. Matej and I. Bajla, "A high-speed reconstruction from projections using direct Fourier method with optimized parameters-An experimental analysis," IEEE Trans. Med. Imag., vol. 9, pp. 421-429, Dec. 1990.

[15] M. Magnusson, P. E. Danielsson, and P. Edholm, "Artefacts and remedies in direct Fourier reconstruction," in Proc. 1992 IEEE Nuclear Science Symp/Medical Imaging Conf, vol. 2, Orlando, FL, 1992, pp. $1138-1140$.

[16] H. Schomberg and J. Timmer, "The gridding method for image reconstruction by Fourier transformation," IEEE Trans. Med. Imag., vol. 14, pp. 596-607, Sept. 1995.

[17] C. W. Stearns, D. A. Chesler, and G. L. Brownell, "Three dimensional image reconstruction in the Fourier domain," IEEE Trans. Nucl. Sci., vol. 34, pp. 374-378, Feb. 1990.

[18] S. Dunne, S. Napel, and B. Rutt, "Fast reprojection of volume data," in Proc. 1st Conf. Visualization in Biomedical Computing, Atlanta, GA, 1990, pp. 11-18.

[19] J. D. O'Sullivan, "A fast sinc function gridding algorithm for Fourier inversion in computer tomography," IEEE Trans. Med. Imag., vol. MI-4, pp. 200-207, Dec. 1985.

[20] H. Sedarat and D. G. Nishimura, "On the optimality of the gridding reconstruction algorithm," IEEE Trans. Med. Imag., vol. 19, pp. 306-317, Apr. 2000.

[21] J. I. Jackson, C. H. Meyer, D. G. Nishimura, and A. Macovski, "Selection of a convolution function for Fourier inversion using gridding," IEEE Trans. Med. Imag., vol. 10, pp. 473-478, Sept. 1991

[22] C. H. Meyer, B. S. Hu, D. G. Nishimura, and A. Macovski, "Fast spiral coronary artery imaging," Magn. Reson. Med., vol. 28, pp. 202-213, 1992.

[23] D. C. Noll, "Multishot rosette trajectories for spectrally selective MR imaging," IEEE Trans. Med. Imaging, vol. 16, no. 4, pp. 372-377, 1997.

[24] J. G. Pipe and P. Menon, "Sampling density compensation in MRI: Rationale and an iterative numerical solution," Magn. Reson. Med., vol. 41, no. 1 , pp. 179-186, 1999.

[25] S. Matej and R. M. Lewitt, "3D-FRP: Direct Fourier reconstruction with Fourier reprojection for fully 3D PET," IEEE Trans. Nucl. Sci., vol. 48, no. 4, pp. 1378-1385, 2001.
[26] S. Matej, M. E. Daube-Witherspoon, and J. S. Karp, "Performance of 3D RAMLA with smooth basis functions on fully 3D PET data," in Proc. 6th Int. Meeting Fully Three-Dimensional Image Reconstruction in Radiology and Nuclear Medicine, R. H. Huesman, Ed., Pacific Grove, CA, 2001, pp. 193-196.

[27] J. A. Fessler and B. P. Sutton, "A min-max approach to the multidimensional nonuniform FFT: Application to tomographic image reconstruction," in Proc. Int. Conf. Image Processing, vol. 1, 2001, pp. 706-709.

[28] _ " "Nonuniform fast Fourier transforms using min-max interpolation," IEEE Trans. Signal Processing, vol. 51, pp. 560-574, Feb. 2003.

[29] B. P. Sutton, D. C. Noll, and J. A. Fessler, "Fast, iterative image reconstruction for MRI in the presence of field inhomogeneities," IEEE Trans. Med. Imag., vol. 22, pp. 178-188, Feb. 2003.

[30] P. E. Kinahan and J. G. Rogers, "Analytic 3D image reconstruction using all detected events," IEEE Trans. Nucl. Sci., vol. 36, pp. 964-968, 1989.

[31] R. M. Lewitt, "Multidimensional digital image representations using generalized Kaiser-Bessel window functions," J. Opt. Soc. Amer. A, vol. 7, no. 10, pp. 1834-1846, 1990.

[32] J. Qi, R. M. Leahy, S. R. Cherry, A. Chatziioannou, and T. H. Farquhar, "High-resolution 3D Bayesian image reconstruction using the microPET small-animal scanner," Phys. Med. Biol., vol. 43, pp. 1001-1013, 1998.

[33] A. J. Reader, P. J. Julyan, H. Williams, D. L. Hastings, and J. Zweit, "EM algorithm resolution modeling by image-space convolution for PET reconstruction," IEEE Trans. Nucl. Sci., vol. 50, no. 5, pp. 1392-1397, 2003.

[34] G. N. Watson, Theory of Bessel Functions, Cambridge, U.K.: Cambridge Univ. Press, 1944.

[35] S. Matej and R. M. Lewitt, "Practical considerations for 3D image reconstruction using spherically symmetric volume elements," IEEE Trans. Med. Imaging, vol. 15, no. 1, pp. 68-78, 1996.

[36] S. Matej, J. A. Fessler, and I. G. Kazantsev, "Fourier-Based Forward and Back-Projectors for Iterative Reconstruction," Dpt. of Radiology, Univ. of Pennsylvania, Philadelphia, Tech. Rep. MIPG303, May 2003

[37] J. A. Fessler, "Penalized weighted least-squares image reconstruction for positron emission tomography," IEEE Trans. Med. Imag., vol. 13, pp. 290-300, June 1994

[38] H. Erdoğan and J. A. Fessler, "Ordered subset algorithms for transmission tomography," Phys. Med. Biol., vol. 44, pp. 2835-2851, 1999.

[39] S. Matej and R. M. Lewitt, "Efficient 3D grids for image reconstruction using spherically symmetric volume elements," IEEE Trans. Nucl. Sci., vol. 42, pp. 1361-1370, Aug. 1995. 